\title{
Effects of stimulus type and level repetition on content-level binding in global/local processing
}

\section{Ronald Hübner* and Rana Kruse}

Department of Psychology, Universität Konstanz, Konstanz, Germany

\section{Edited by:}

Paul Sajda, Columbia University, USA

Reviewed by:

Andreas Keil, University of Florida, USA

Karla K. Evans, Harvard Medical

School, USA

Anastasia V. Flevaris, University of

California, USA

*Correspondence:

Ronald Hübner, Fachbereich

Psychologie, Universität Konstanz,

Fach D29, D-78457 Konstanz,

Germany.

e-mail: ronald.huebner@uni-konstanz.de

The processing and representation of hierarchical objects not only involves the identification of information at the different levels, but also the binding of the identified content to its respective level. Whereas identification is well understood, little is known about content-level binding (CLB). In a recent study, however, it has been shown that attentional priming of certain spatial frequencies is advantageous for this binding. Therefore, the present study investigated effects of related factors on the binding process, namely stimulus type (filled or outlined hierarchical letters), stimulus-type repetition, and target-level repetition. The results show that CLB was improved for outlined stimuli and after target-level repetition, whereas stimulus-type repetition had no effect. The data suggest that hierarchical stimuli are mentally represented by abstract level categories and that content is linked to these categories by means of level-specific and identity-specific spatial-frequency information.

\section{Keywords: global/local processing, feature binding, conjunction errors, visual-field effects}

\section{INTRODUCTION}

Most organic or manmade objects such as trees or cars have a hierarchical structure, i.e., their global form is composed of various local components. An important and widely investigated question is how such objects are perceived and mentally represented. Many studies, mostly using hierarchical letters (see Figure 1) as stimuli (Navon, 1977), have shown that it is possible or sometimes even advantageous to select only the information at a specific target level. But how is this level-specific selection achieved? Originally, it was thought that separate information channels or pathways for each level transmit corresponding information during perception (e.g., Lovegrove and Pepper, 1994; May et al., 1995; Lamb and Yund, 1996), and that individuals select level-specific information by attending to the output of the respective channel.

Hübner and Volberg (2005), however, have questioned that selection proceeds this way. In their "content-level binding" (CLB) theory they proposed that, at a first stage of processing, the contents of the different levels in a hierarchical object are identified and represented independently from their respective level. Consequently, information selection requires active binding of a particular level to its content, which is assumed to take place at a late stage. To test their CLB theory, Hübner and Volberg (2005) conducted a series of experiments in which participants had to report the identity of a target letter at a pre-specified level in a hierarchical stimulus. Each hierarchical stimulus contained two out of four possible letters. Moreover, each stimulus was masked shortly after its presentation to impair CLB. It was hypothesized that, if the two letters are identified at an early stage, the impaired binding process should produce conjunction errors. Indeed, participants mistakenly reported the letter at the non-target level of the stimulus more frequently than the other two letter identities not present in the display. The result suggests that the letter at the irrelevant level had been erroneously linked to the target level, which is compatible with the idea that the contents of a hierarchical object are identified independently from their level (for details see Hübner and Volberg, 2005).
Previous studies had already shown that binding between features like form, color, or spatial location is necessary for the construction of mental object representations (Treisman and Gelade, 1980; Treisman and Schmidt, 1982). A relatively novel idea, though, is that binding also occurs between the contents and levels of a hierarchical object. Accordingly, little is known about the corresponding mechanism, yet. The objective of the present study was, therefore, to gain further insight into this mechanism by examining factors that potentially affect the efficiency of CLB. Unfortunately, because binding presupposes identification, the observable rate of conjunction errors depends on the efficiency of both binding and identification. Consequently, one cannot decide whether a factor affected the binding process or not by simply inspecting the rates of conjunction errors. Rather, one needs a method that allows one to separate factor effects on binding from those on identification.

A practical solution to this problem has recently been demonstrated by Flevaris et al. (2010) in a study in which they investigated whether CLB can be improved by spatial-frequency priming. To separate the priming effect on binding from those on identification they also considered functional asymmetries of the cerebral hemispheres. It is well known that the left hemisphere (LH) and the right hemisphere (RH) are specialized for local and global processing, respectively (e.g., Van Kleeck, 1989; Yovel et al., 2001; Hübner and Malinowski, 2002; Hübner et al., 2007; Hübner and Studer, 2009). This also holds for CLB. Hübner and Volberg (2005) have shown that responses to the local target level yielded more conjunction errors for stimuli presented to the left visual field (LVF) than for stimuli in the right visual field (RVF), whereas the opposite relation held for the global target level. From the fact that such visual-field (VF) effects were absent for neutral stimuli that did not require CLB, it can be concluded that the cerebral hemispheres have the same capacity for letter identification, but differ in their binding efficiency. The LH is more efficient at binding content to the local level whereas the $\mathrm{RH}$ is superior in binding information to the global level. 
This hemispheric asymmetry is also supported by neurophysiological results. Besides electrophysiological studies (Malinowski et al., 2002; Volberg and Hübner, 2004), also neuropsychological studies provide some evidence. Doricchi and Incoccia (1998), for instance, described a patient with RH damage. She had no problems with seeing a global shape, but only when there were no relevant local shapes. This indicates that her identification performance was intact, but that her binding mechanism was impaired.

In view of these hemispheric asymmetries, Flevaris, et al. (2010) assumed that the non-specialized hemisphere for a given level should benefit more from a factor that improves the binding process for that level than the specialized one. Consequently, the corresponding VF effects in conjunction errors should be reduced. In contrast, a factor that merely improves identification should reduce the rate of conjunction errors equally for both hemispheres. In Flevaris, et al.'s (2010) experiment participants had to identify the letter at the target level of a hierarchical stimulus. Critically, prior to the identification task, they categorized the orientation of a specific spatial-frequency component in a compound sinusoidal grating on each trial. The results showed that the categorization of a high-spatial-frequency component reduced the subsequent VF effects in conjunction errors for the local target level, whereas responding to a low-spatial-frequency component reduced the subsequent VF effects for the global target level. From these results Flevaris, et al. (2010) concluded that “....attentional selection of spatial-frequency information plays a key role in binding elements of hierarchical displays to the levels at which they occur" (p. 430).

Encouraged by these results we further examined effects of spatial frequency and related factors on CLB. One hypothesis was that CLB also improves when the target level repeats between subsequent trials compared to level shifts. From response-time studies it is known that level repetition has a positive effect on performance, which is presumably due to attentional level priming (e.g., Robertson et al., 1993; Robertson, 1996; Hübner, 2000). Therefore, it was conceivable that CLB also benefits from the priming.

Furthermore, we reasoned that the specific spatial-frequency content of hierarchical letters may affect content binding. Therefore, we employed filled and outlined versions of the stimuli. Compared to filled letters, outlined letters have less spectral power in the low-spatialfrequency range but more spectral power in the high-spatial-frequency range (for example stimuli and their spectra see Figure 1). Although Figure 1 suggests that this difference is small in the present case, the two stimulus types can nevertheless produce substantial differences in performance (Hübner, 1997). Usually, the proportion of low- and high-spatial-frequencies determines, at least to some extent, the relative salience of levels. It has been argued that this is due to the different characteristics of magnocellular and parvocellular pathways, which transmit low- and high-spatial-frequency information, respectively (e.g., Hughes et al., 1996). Typically, the transmission is faster in the magnocellular pathway. Thus, if low spatial frequencies have more power and if this is favorable for attending to the global level, then the binding may be improved for this level. As a consequence, the VF effects for the global level should be smaller for filled than for outlined stimuli. An analogous reasoning predicts the opposite for the local level.

The specific spatial-frequency content of the stimuli could also have another effect on CLB. According to the double filtering by frequency (DFF) theory (Ivry and Robertson, 1998), the spatial frequencies of a hierarchical stimulus are first parsed and then linked to the levels. Thus, if the separation of the spatial-frequency ranges corresponding to the two levels is easier for one stimulus type than for another, then this may also result in more efficient CLB for the one type. Moreover, spatial-frequency parsing and, consequently, CLB could also be enhanced when the current spatial-frequency ranges are the same as those on the last trial. Whether this is the case should be examined by analyzing effects of stimulus-type repetition.

The considerations of stimulus level and spatial-frequency demonstrate that these factors are related in some way. However, there are also results indicating that level repetition and spatial-frequency repetition produce independent effects (e.g., Robertson, 1996; Kim et al., 1999; Lamb et al., 1999). Unfortunately, up to now little is known about the details of the relation between these factors. Accordingly, our hypotheses about effects of stimulus level and spatial frequency on CLB must remain relatively vague.

In both experiments of this study we applied the masking paradigm (Hübner and Volberg, 2005). That is, a hierarchical letter was presented and masked after a certain stimulus-mask interval (SMI). Two different SMIs were randomized in each block of trials. Based on prior experience, interval lengths were chosen that produce an appropriate number of conjunction errors for each participant. Usually, the rate of conjunction errors decreases with an increasing SMI. Because this effect was of no interest for the present objective, SMI was not included as factor in the data analyses.

\section{EXPERIMENT 1}

In our first experiment we did not distinguish between prime and main stimulus. Rather, all stimuli were masked hierarchical letters. There were four possible letter identities, and the identities of the two letters present in a stimulus were always different. Participants were instructed to identify the letter at the pre-specified target level. When participants falsely reported the letter at the non-target level, their response was categorized as conjunction error, and when they reported a letter not present in the stimulus, it was categorized as non-conjunction error. Concerning conjunction errors it is possible that the letter at the nontarget level is named simply by guessing when none of the letters in the stimulus was identified. Thus, to be sure that there were "real" erroneous conjunctions, we tested their rate against the chance level of 1/3.

To measure VF effects, the stimuli were randomly presented either to the LVF or to the RVF. Accordingly, VF effects for global/ local processing are typically indicated by a significant interaction between target level and VF. In the present case, this means that for the local target level more conjunction errors should occur for LVF-stimuli than for RVF-stimuli, whereas the opposite should hold for the global target level.

The factors stimulus type and target level were also randomized within each block of trials. Consequently, the effects of level repetition had to be assessed by examining sequential effects. For this purpose the trials were categorized as repetition trial or shift trial after the experiment. An analogous procedure was performed for the factor stimulus-type repetition. Thus, when the repetition of stimulus type or of target level improves CLB, then the VF effects should be reduced on repetition trials compared to shift trials. Moreover, when stimulus type as such modulates the efficiency of CLB, then the VF effects should vary with this factor. 

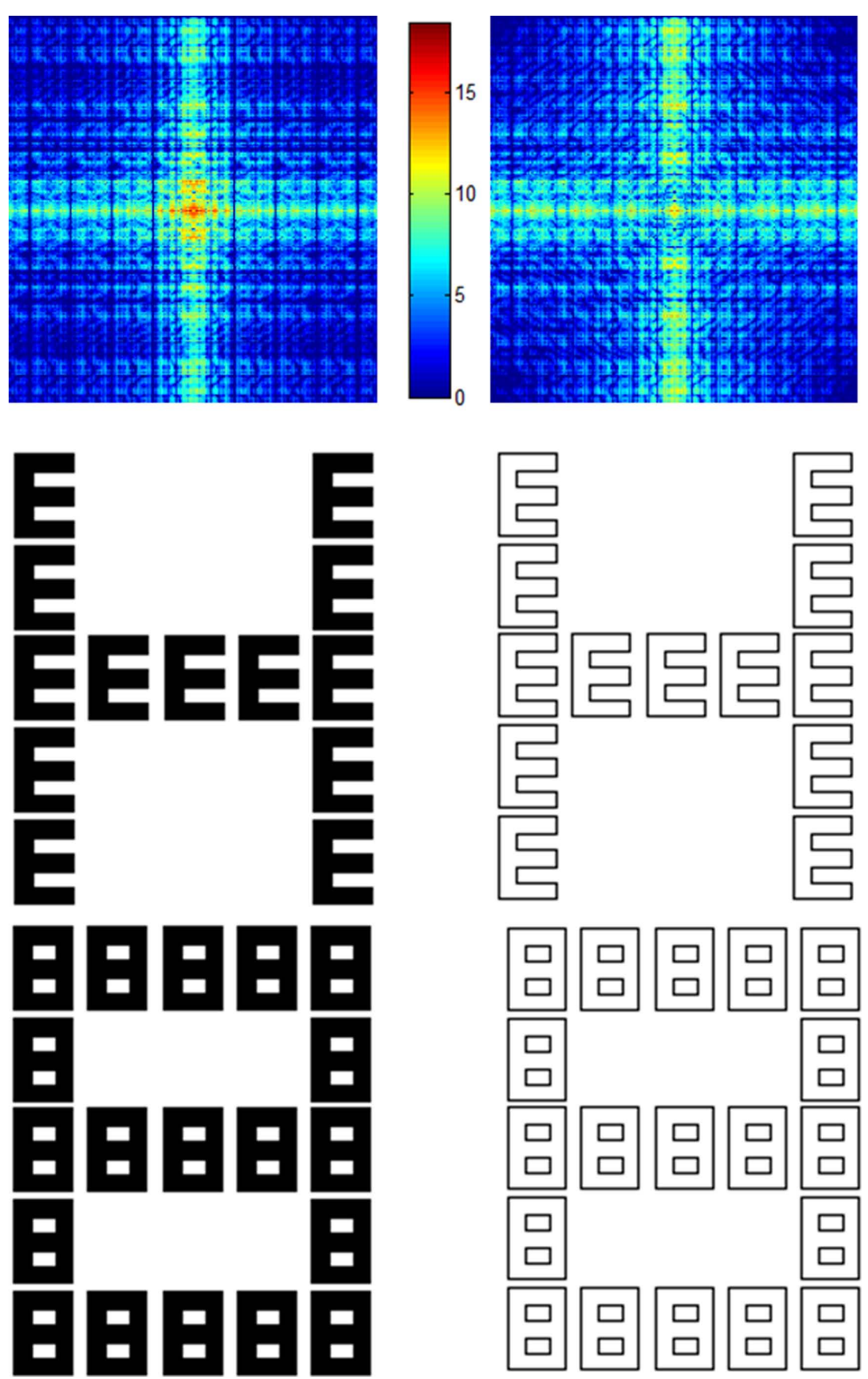

FIGURE 1 | In the middle row examples of filled (on the left) and outlined (on the right) hierarchical letters are shown. The corresponding masks can be seen below the stimuli. In the experiments stimuli and masks were presented in white on a black background. The top row represents the corresponding log-power spectra of the example stimuli. In these plots, spatial-frequency increases from the center to the borders.

\section{METHOD}

\section{Participants}

Twenty-eight students (mean age of 22.2 years; seven male) from the Universität Konstanz, Germany, participated in the experiment. All had normal or corrected-to-normal vision, were right-handed by self-report, and were paid $15 €$ for their participation.

\section{Apparatus and stimuli}

Stimuli were presented on a $18^{\prime}$ color-monitor with a resolution of $1280 \times 1024$ pixels and a refresh rate of $60 \mathrm{~Hz}$. Participants responded by pressing one of four buttons of a computer keyboard. Stimulus presentation as well as response registration was controlled by the same personal computer (PC). 
Stimuli were outlined and filled hierarchical letters (for examples see Figure 1). Each stimulus was constructed from two out of four different letters (A, S, H, E). The size of the global letters was $4.48^{\circ}$ of visual angle horizontally and $5.72^{\circ}$ vertically. The respective size of the local letters was $0.72^{\circ} \times 1.08^{\circ}$. Depending on the stimulus type, the local letters were constructed by outlines or were additionally filled. Stimuli were presented in white on a black background either to the LVF or to the RVF at an eccentricity of $2.82^{\circ}$ (from the midline of the screen to the center of the stimulus).

\section{Procedure}

Participants were seated at a viewing distance of approximately $60 \mathrm{~cm}$ in front of the screen. A trial started with the presentation of a cue (the letter $l$ or $g$ to indicate a local or global target level, respectively) at the center of the screen for $300 \mathrm{~ms}$, followed by a blank screen for $100 \mathrm{~ms}$. After the presentation of a fixation cross for $300 \mathrm{~ms}$ and a subsequent blank screen of $50 \mathrm{~ms}$ the stimulus was presented for $32 \mathrm{~ms}$ either to the LVF or to the RVF. The SMI was 48 or $80 \mathrm{~ms}$. VF, target level, stimulus type, and SMIs were randomized across trials. The mask was presented on both VFs and remained present on the screen until the response occurred. The task was to identify the letter at the cued level. Participants responded by pushing one of four response buttons of the keyboard (each corresponding to a certain letter). The button-to-letter mapping was varied across participants to counteract any response biases. Participants were advised that each stimulus contained two different letters, so that, if they identified the letter at the non-target level, they should not report this letter but guess one of the remaining letters. At the end of each block a feedback screen was displayed to inform the participants about their error rate on the current block. If the error rate exceeded $50 \%$ the participants were asked to increase their effort.

After some training, 12 blocks of 96 trials for each participant were run in a single 2 -h session, including a 15 -min break. This resulted in 72 trials per condition.

\section{RESULTS}

Conjunction errors occurred on $10.6 \%$ of the trials. Their proportion on the overall error rate $(18.2 \%)$ was $59.2 \%$, which was significantly greater than the maximally possible guessing rate of $1 / 3, F(1$, $27)=200, p<0.001$. First, the rates of conjunction errors, of nonconjunction errors, and the response times were entered into separate three-factor ANOVAs on the within-participants factors stimulus type (outlined or filled), target-level (global or local), and VF (LVF or RVF). In additional ANOVAs sequential repetition effects were analyzed.

\section{Conjunction errors}

The main effect of VF was significant, $F(1,27)=8.92, p<0.01$. More errors occurred for RVF-stimuli than for LVF-stimuli (11.2 versus $10.0 \%)$. However, as expected, VF interacted with target level, $F(1$, $27)=21.5, p<0.001$. For the global target level more conjunction errors occurred for RVF-stimuli than for LVF-stimuli (12.9 versus $8.3 \%$ ), whereas the opposite held for the local target level (9.5 versus $11.7 \%)$. There was also a significant two-way interaction between level and stimulus type, $F(1,27)=41.5, p<0.001$. For filled stimuli more conjunction errors occurred for the global target level than for the local target level (12.0 versus $8.7 \%$ ), whereas the opposite was true for the outlined stimuli (9.2 versus $12.5 \%$ ).

\section{Non-conjunction errors}

The main effect of stimulus type, $F(1,27)=55.8, p<0.001$, and that of level were significant, $F(1,27)=13.2, p<0.01$. More errors occurred for outlined stimuli than for filled stimuli (9.1 versus $6.1 \%$ ), and for responses to the local level than for those to the global level (10.6 versus 4.6\%). However, there was also a significant two-way interaction between these factors, $F(1,27)=31.0$, $p<0.001$. Whereas for the global target level the error rates were similar for both stimulus types, they differed substantial for the local target level (global: filled 4.3\% versus outlined 4.9\%; local: filled $7.8 \%$ versus outlined $13.3 \%$ ). There was also a two-way interaction between stimulus type and VF, $F(1,27)=9.79, p<0.01$. For filled stimuli more errors occurred for RVF-stimuli than for LVFstimuli (6.4 versus 5.7\%), whereas the relation was reversed for outlined stimuli ( 8.4 versus $9.8 \%$ ). Finally, VF interacted with target level, $F(1,27)=15.2, p<0.001$. For the global target level more errors occurred for RVF-stimuli than for LVF-stimuli (5.5 versus $3.7 \%)$, whereas for the local target level the relation was reversed (9.4 versus $11.8 \%)$.

\section{Response times}

The main effect of target level was significant, $F(1,27)=10.7$, $p<0.01$. However, there was also a significant interaction between target level and VF, $F(1,27)=19.5, p<0.001$, indicating faster responses to LVF-stimuli than to RVF-stimuli on the global level (796 versus $835 \mathrm{~ms}$ ), whereas the pattern was reversed for the local level (894 versus $872 \mathrm{~ms}$ ). There was also a two-way interaction between target level and stimulus type, $F(1,27)=21.7, p<0.001$. The difference between response latencies to the global and the local level was smaller for filled (global $831 \mathrm{~ms}$ versus local $872 \mathrm{~ms}$ ), than for outlined stimuli (global $800 \mathrm{~ms}$ versus local $895 \mathrm{~ms}$ ).

\section{Repetition effects}

For analyzing the effects of level repetition and stimulus-type repetition and their interaction with the relevant other factors each trial was categorized as repetition trial or as shift trial. Corresponding mean conjunction-error rates and mean latencies were entered into separate four-factor ANOVAs on the within-participants factors stimulus-type repetition (repetition, shift), level repetition (target-level repetition or target-level shift), target level (global or local), and VF (LVF or RVF).

In the conjunction errors, there was a main effect of level repetition, $F(1,27)=12.2, p<0.01$, with fewer conjunction errors for a repeated than for a shifted level (8.58 versus $12.1 \%)$. VF effects were neither modulated by level repetition (see also in Figure 2) nor by stimulus-type repetition.

Likewise, a main effect of level repetition for response times, $F(1$, $27)=33.3, p<0.001$ revealed shorter latencies for repeated than for shifted target levels ( 813 versus $866 \mathrm{~ms}$ ). Neither level repetition (see also in Figure 2) nor stimulus-type repetition interacted significantly with target level or VF.

\section{DISCUSSION}

The results show that our method successfully generated a high rate of conjunction errors. Moreover, there were VF effects in the expected directions not only for conjunction errors, but also for non-conjunction errors, and for response times. Although in some experiments the interaction between VF and target level was 


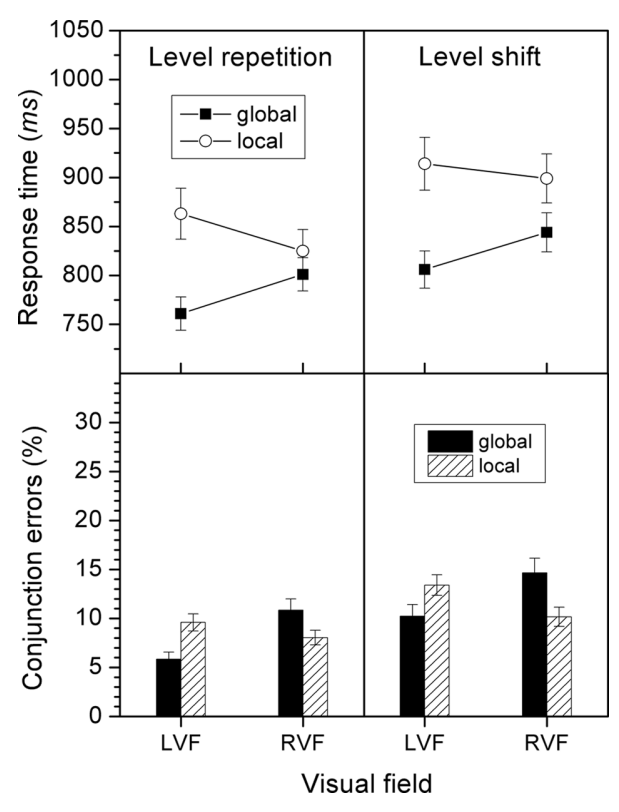

FIGURE 2 | Response times and conjunction errors for level-repetition and level-shift conditions in Experiment 1. The size of the VF effects for a given level are reflected by the difference in height between the respective bars or data points for the LVF and RVF.

absent in the non-conjunction errors (Experiment 1 in Hübner and Volberg, 2005; Flevaris et al., 2010), its occurrence is not unusual (Experiments 2 and 3 in Hübner and Volberg, 2005) and in line with the CLB theory. Clearly, impaired CLB can also lead to guessing, which then increases the rate of non-conjunction errors (for details see Hübner and Volberg, 2005). Therefore, it is not surprising if the non-conjunction errors mimic to some extent the effects of conjunction errors. In any case, because non-conjunction errors are uninformative for our objective we will concentrate our considerations on the conjunction errors and response times.

With respect to our main questions, the data analysis revealed that stimulus-type produced several effects. First, stimulus-type modulated the global advantage in RT (Hübner, 1997; Miller and Navon, 2002). Although responses were generally faster for the global than for the local target level, the difference was more pronounced for the outlined stimuli. More specifically, compared to the filled stimuli, outlined stimuli led to faster RTs for the global level and to slower RTs for the local level. Likewise, outlined stimuli produced fewer conjunction errors for the global level and more conjunction errors for the local level than filled stimuli. These relations are contrary to what we had expected, given the spatial-frequency difference between the stimulus types (e.g., Lamb, et al., 1999). They suggest that inferences from studies with unmasked stimuli are not readily applicable to experiments with masked stimuli.

In any case, these results demonstrate that the difference in spatial-frequency between the stimulus types had an effect on the relative level dominance. However, it did not modulate the VF effects. Thus, the obtained pattern of results seems to indicate that stimulus-type influenced the rate at which the letter at the target level was identified, but not the efficiency with which the identified letter was linked to its level.
Concerning the repetition of stimulus type, our results indicate that it had no effect. In contrast, level repetition not only speeded up the responses but also produced fewer conjunction errors. However, level repetition had no effect on the VF effects.

Taken together, the data of this experiment do not support the idea that stimulus type, stimulus-type repetition, or level-repetition affects CLB. Does this mean that all of these factors have generally no effect in this respect? Instead of drawing this conclusion, we hypothesized that our method might not have been appropriate. Flevaris et al. (2010) primed the target level with specific unmasked stimuli, whereas in the present experiment all stimuli were masked. Perhaps the positive effects of repetition were absent, because the mask destroyed all level-specific priming effects, or because all stimuli were presented laterally. Moreover, in Flevaris et al.'s (2010) study target level and level repetition were blocked, whereas all factors were randomized in the present experiment. To see whether these procedural differences are crucial, we conducted a second experiment.

\section{EXPERIMENT 2}

The aim of our second experiment was the same as that of the first one. We again wanted to test whether stimulus type, stimulus-type repetition, and level-repetition improve the binding of content and level during the processing of hierarchical stimuli. Here, however, the employed procedure was different from that in the previous experiment. Instead of exclusively using masked stimuli, an unmasked prime stimulus was presented before the main stimulus and at the center of the screen, analogous to the procedure in Flevaris et al.'s (2010) study. Different from that study, though, prime stimuli were also hierarchical letters, and the task of the participants for these stimuli was, as for the main stimuli, the identification of the letter at a pre-specified target level. Prime stimuli and main stimuli could be filled or outlined hierarchical letters (see Figure 1).

Moreover, in this experiment main-stimulus type, primestimulus type, target level, stimulus-type repetition, and level repetition were blocked. There was one group of participants for each of the four possible combinations of main-stimulus type and prime-stimulus type. Target-level repetition was varied within each participant. In some blocks of trials the target level repeated from the prime task to the main task, whereas in other blocks it changed.

\section{METHOD}

\section{Participants and procedure}

Forty-eight students (mean age of 23.2 years; 16 male) from the Universität Konstanz participated in the experiment and were randomly assigned to one of four groups (see below). All had normal or corrected-to-normal vision, were right-handed by self-report, and were paid $15 €$ for their participation.

The method was similar to Experiment 1. The main difference was that two tasks were required on each trial: a prime task and a main task. For performing the prime task the participants had to identify the letter at the target level of a centrally presented hierarchical letter. They were instructed to respond as quickly and as accurately as possible. The same four buttons as for the main task were used for the prime task, and the button-to-letter mapping varied across participants. The main task was the same as in Experiment 1. Prime stimuli and main stimuli could be filled hierarchical letters or outlined hierarchical letters (see Figure 1). There was one group 
of participants for each combination of prime-stimulus type and main-stimulus type. Accordingly, two groups had filled hierarchical letters as main stimuli and two groups had outlined hierarchical stimuli. And for each of these two groups one group had filled stimuli as primes and the other had outlined stimuli as primes. Each trial started with the central presentation of a cue for $300 \mathrm{~ms}$ indicating the target level of the prime task (the letter $l$ or $g$ for local or global target level, respectively). After a black blank screen of $100 \mathrm{~ms}$ duration the prime stimulus was presented at the center of the screen and remained present until the response. Immediately after the response the cue for the main stimulus was presented at the center of the screen. The procedure for the main task was identical to that in Experiment 1. After each block of trials feedback on the performance in each task was provided. When more than $10 \%$ errors had occurred in the prime task, the participants were urged to respond more carefully. Target levels for the two tasks on a trial were blocked in all four combinations: global-local, local-global, local-local, global-global. After some training, four blocks á 72 trials of each type were administered in sequential order, which was balanced across participants. This resulted in 48 trials per condition. All trials were run in a 2 -h session including a 15 -min break.

\section{RESULTS}

\section{Responses to first stimulus}

Mean error rates and mean response times of correct responses to the first stimulus were subjected to separate four-factor ANOVAs on the between-participants factor prime type (outlined or filled), prime condition (type repetition or type shift), and the withinparticipants factors target level (global or local), and level repetition (target level repetition or target level shift).

On average, the error rate was $2.9 \%$. The main effects of the factors prime condition, $F(1,44)=8.58, p<0.01$, target level, $F(1$, $44)=5.94, p<0.05$, and level repetition, $F(1,44)=15.6, p<0.001$, were significant. More errors occurred when prime and stimulus were of different types than when they were of the same type (3.6 versus $2.1 \%$ ). Concerning the target level, there were more errors for responses to the local level than for those to the global level (3.3 versus $2.5 \%$ ). Finally, accuracy was higher when the target-level repeated than when it shifted (2.2 versus $3.5 \%$ ).

For the response times there were significant main effects of target level, $F(1,44)=61.5, p<0.001$, and of level repetition, $F(1$, $44)=185, p<0.001$. Letters at the local level were identified faster than those at the global level ( 849 versus $950 \mathrm{~ms}$ ), and responses in blocks with target-level repetitions were faster than those in blocks with target-level shifts ( 815 versus $983 \mathrm{~ms}$ ).

\section{Responses to the second stimulus}

The mean rate of conjunction errors was $12.2 \%$, at a total mean error rate of $22.2 \%$. Thus, the proportion of conjunction errors was $54.9 \%$ which was significantly greater than the maximally possible guessing rate of $1 / 3, F(1,44)=223, p<0.001$.

The mean rates of conjunction errors and of non-conjunction errors, and the mean response times were entered into separate fivefactor ANOVAs on the between-participants factor stimulus type (outlined or filled) and prime condition (type repetition or type shift) and the within-participants factors level repetition (target-level repetition or target-level shift), target level (global or local), and VF (LVF or RVF).
Conjunction errors. The main effect of level repetition was significant, $F(1,44)=80.1, p<0.001$, indicating that fewer errors occurred under level repetition than under level shifting (10.0 versus $14.4 \%)$. There were also significant main effects of target level, $F(1,44)=27.6, p<0.001$, and of VF, $F(1,44)=6.25, p<0.05$. More errors occurred for the local than for the global target level (14.8 versus $9.7 \%$ ), and for LVF-stimuli, compared to RVF-stimuli (12.9 versus $11.5 \%)$. However, both factors interacted in the usual way, $F(1,44)=149, p<0.001$. For the global target level, errors occurred more frequently for RVF-stimuli than for LVF-stimuli (12.6 versus $6.7 \%$ ), whereas the opposite held for the local level (10.3 versus $19.2 \%$ ). Most importantly for the present objective, there was also a significant three-way interaction between target level, VF, and level repetition, $F(1,44)=20.7, p<0.001$. Under level repetition the VF effects were smaller (global: RVF 10.2\% versus LVF 5.7\%; local: RVF 8.2\% versus LVF 15.9\%) than under level shifting (global: RVF 15.1\% versus LVF 7.7\%; local: RVF $12.4 \%$ versus LVF 22.5\%). See also Figure 3.

There was also a significant three-way interaction between target level, VF, and stimulus type, $F(1,44)=24.1, p<0.001$. For outlined stimuli the VF effects were smaller (global: RVF 10.2\% versus LVF 6.1\%; local: RVF $12.3 \%$ versus LVF $17.1 \%$ ) than for filled stimuli (global: RVF 15.1\% versus LVF 7.3\%; local: RVF 8.4\% versus LVF $21.3 \%)$. See also Figure 4. However, the VF effects did not vary with the prime condition, as indicated by the non-significant threeway interaction between prime condition, $\mathrm{VF}$, and target level, $F(1$, 44) $=0.813, p=0.372$.

Non-conjunction errors. The main effect of level repetition was significant, $F(1,44)=13.5, p<0.001$, indicating that level-repetitions produced fewer errors than level shifts ( 9.2 versus $10.8 \%$ ). There

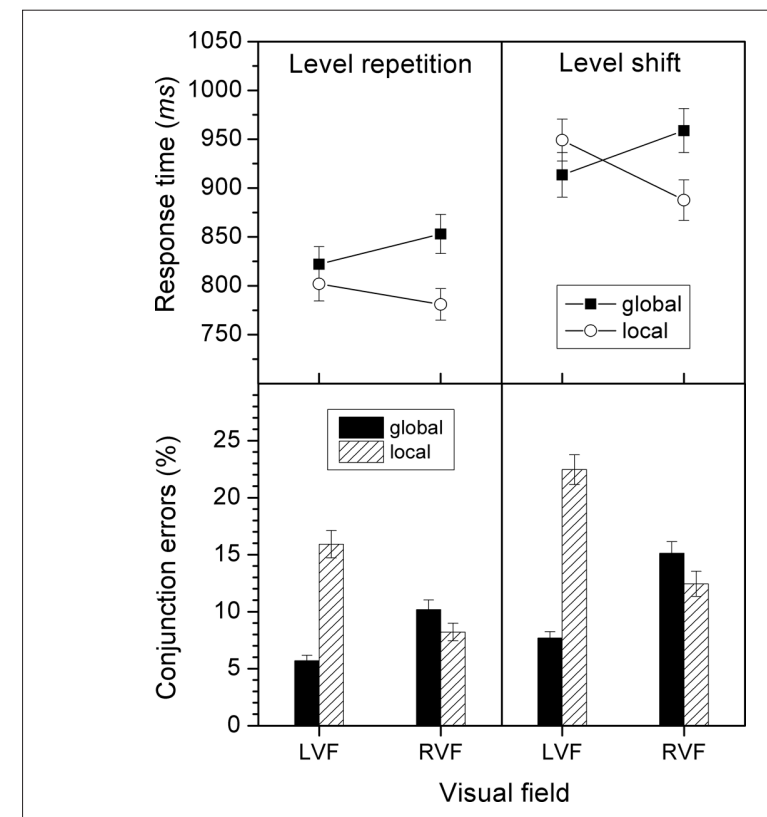

FIGURE 3 | Response times and conjunction errors for level repetitions and level shifts in Experiment 2. The size of the VF effects for a given level are reflected by the difference in height between the respective bars or data points for the LVF and RVF. 


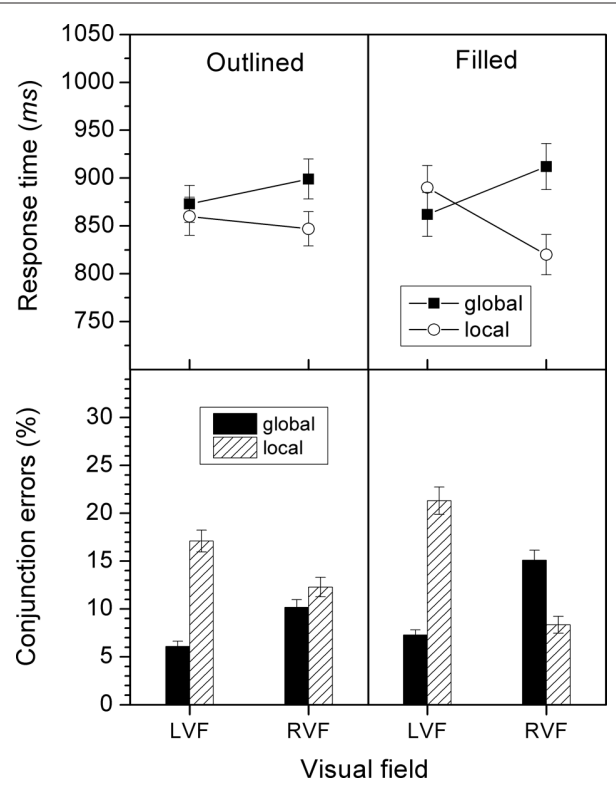

FIGURE 4 | Response times and conjunction errors for the two stimulus types in Experiment 2. The size of the VF effects for a given level are reflected by the difference in height between the respective bars or data points for the LVF and RVF.

were also significant main effects of target level, $F(1,44)=30.4$, $p<0.001$, and of VF, $F(1,44)=9.24, p<0.01$. More errors occurred for the local target level than for the global one (11.8 versus $8.1 \%$ ), and for LVF-stimuli, compared to RVF-stimuli (10.7 versus 9.3\%). However, both factors interacted significantly, $F(1,44)=76.4$, $p<0.001$. For the global target level, errors occurred more frequently for RVF-stimuli than for LVF-stimuli (9.1 versus 7.2\%), whereas the opposite held for the local level (9.4 versus 14.2\%). There was also a two-way interaction between stimulus type and target level, $F(1,44)=7.39, p<0.001$. For outlined stimuli the difference between the target levels was larger than for filled stimuli (outlined: global 7.9\% versus local 13.4\%; filled: global $8.4 \%$ versus local $10.2 \%)$. Moreover, there was a significant three-way interaction between target level, VF, and level repetition, $F(1,44)=5.24$, $p<0.05$. For the global level, level repetitions did not modulate the VF effect (repetition: RVF 8.4\% versus LVF 6.4\%; shift: RVF 9.9\% versus LVF 7.9\%), whereas for the local level the VF effect was smaller under level repetition than under level shifting (repetition: RVF 9.0\% versus LVF 12.8\%; shift: RVF 9.9\% versus LVF $15.7 \%)$. There was also a significant three-way interaction between target level, VF, and stimulus type, $F(1,44)=9.96, p<0.01$. For outlined stimuli the VF effects were smaller (global: RVF 8.5\% versus LVF 7.4\%; local: RVF 11.8\% versus LVF 15.0\%) than for filled stimuli (global: RVF 9.8\% versus LVF 7.0\%; local: RVF 7.0\% versus LVF 13.4\%).

Response times. The main effect of target level, $F(1,44)=5.93$, $p<0.05$, was significant. Responses to the local target level were faster than those to the global one ( 855 versus $887 \mathrm{~ms}$ ). There was also a significant main effect of level repetition, $F(1,44)=134.1$, $p<0.001$, indicating that responses were faster when the target level repeated than when it shifted ( 815 versus $927 \mathrm{~ms}$ ). However, there was also an interaction between target level and VF, $F(1,44)=53.0$, $p<0.001$. For the global target level, responses were faster for LVFstimuli than for RVF-stimuli (868 versus $906 \mathrm{~ms}$ ), whereas the opposite held for the local target level ( 876 versus $834 \mathrm{~ms}$ ). Most important for the present objective, there was also a significant three-way interaction between target level, VF, and level repetition, $F(1,44)=17.9, p<0.001$. Under level repetition the VF effects were smaller (global: RVF 853 ms versus LVF 822 ms; local: RVF $781 \mathrm{~ms}$ versus LVF $802 \mathrm{~ms}$ ) than under level shifting (global: RVF 959 ms versus LVF 913 ms; local: RVF 888 ms versus LVF 949 ms). See also Figure 3.

Additionally, there was a significant three-way interaction between target level, VF, and stimulus type, $F(1,44)=13.4, p<0.001$. For filled stimuli the VF effects were larger (global: RVF $912 \mathrm{~ms}$ versus LVF 862 ms; local: RVF 821 ms versus LVF $890 \mathrm{~ms}$ ) than for outlined stimuli (global: RVF 900 ms versus LVF 873 ms; local: RVF 848 ms versus LVF $861 \mathrm{~ms}$ ). See also Figure 4. The VF effects did not depend on the prime condition, as indicated by the nonsignificant three-way interaction between target level, VF, and prime condition, $F(1,44)=0.021, p=0.886$.

\section{DISCUSSION}

In many respects the data are similar to those in our first experiment. For instance, the VF effects were again in the expected direction. Also target-level repetition had a positive effect on performance. Responses were faster and there were fewer errors when the target level repeated from the prime task to the main task, compared to when it shifted. Stimulus-type repetition had again no effect.

However, there were also important differences compared to our first experiment. In the present experiment neither stimulustype produced a global advantage, which was presumably due to the fact that target level remained constant in a block of trials. Interestingly, though, in this experiment the type of the mainstimulus (filled versus outlined) modulated the VF effects (see Figure 4). In response time as well as in conjunction errors the VF effects were larger for the filled stimuli than for the outlined stimuli. These results suggest that under the present conditions CLB was easier with outlined stimuli than with filled stimuli. With respect to stimulus-type repetition, there was a positive repetition effect in error rates for the prime but not for the main stimulus. Thus, this factor seems not to have affected CLB. However, this time the repetition of target level reduced the corresponding VF effect (see Figure 3), which indicates improved eficiency of CLB.

\section{GENERAL DISCUSSION}

The aim of the present study was to examine the mechanisms involved in the binding of the contents of a hierarchical object to their respective level. According to the CLB theory (Hübner and Volberg, 2005) such CLB is necessary, because the contents of a hierarchical object are identified and represented independently of their level at an early stage of processing. This idea is supported by results showing that, if stimulus processing is impaired by a mask, conjunction errors between levels and contents occur, i.e., content is often linked to a wrong level. The present results demonstrate once more that conjunction errors make up a high proportion on all errors. 
Up to now, however, little is known about the mechanisms involved in CLB. One characteristic that has already been identified is that the cerebral hemispheres differ in their binding capacity. The RH is better at binding content to the global level, whereas the LH is more efficient for the local level (Hübner and Volberg, 2005). Flevaris et al. (2010) utilized these hemispheric asymmetries to demonstrate a further property of the binding mechanism. They have shown that responding to a low-spatial-frequency component of a compound sinusoidal grating reduced the LH disadvantage for the global target level on the next trial, whereas responding to a high-spatial-frequency component reduced the $\mathrm{RH}$ disadvantage for the local target level. Thus, it seems that the selection and processing of information within a certain spatial-frequency range improves the CLB process for the associated target level. Moreover, this holds especially for the respective inferior cerebral hemisphere. Accordingly, Flevaris et al. (2010) concluded that the attentional selection of spatial-frequency plays a key role for CLB.

Encouraged by Flevaris et al.'s (2010) study we investigated whether other factors also affect CLB. One of these factors was stimulus type, which was varied by employing two different stimulus types: outlined and filled hierarchical stimuli (see Figure 1). Outlined stimuli have less spectral power in the low-spatialfrequency range than filled stimuli, but more relative power in the high-spatial-frequency range. If the selection of spatial frequency is involved in the binding process, then it is conceivable that the specific spatial-frequency composition of a stimulus has also an effect on the binding process. For instance, the relative power in the lower and upper spatial-frequency bands usually determines the relative salience of the stimulus levels, which, in turn, controls how much attention is automatically allocated to the target level. Thus, if the amount of attention available for the target level influences the efficiency of CLB, then different stimulus-types should produce different VF effects in the conjunction errors.

Another possibility was that the specific spatial-frequency composition of a stimulus-type determines the difficulty with which the frequencies can be parsed into bands corresponding to the two hierarchical levels (Ivry and Robertson, 1998). If the efficiency of content-level binding depends on this difficulty in some way, then it is conceivable that the VF effects in conjunction errors might be smaller for a stimulus type that can be parsed more easily. Furthermore, it was conceivable that the parsing process also benefits from the repetition of a stimulus type, which should lead to reduced VF effects on type repetition trials.

A further factor that was investigated was target-level repetition. Various studies have shown that performance is improved when the target level repeats (Robertson, et al., 1993; Robertson, 1996; Lamb, et al., 1999; Hübner, 2000). Thus, if target-level repetition also improves CLB, then the VF effects in the conjunction errors should be reduced on level-repetition trials.

In our first experiment stimulus type and target level were randomized. Moreover, all stimuli were masked. The data analysis revealed that stimulus-type modulated the relative strength of the levels. Although the performance was generally better for the global than for the local target level, the difference was larger for the outlined stimuli than for the filled stimuli. However, neither stimulus type nor its repetition affected the VF effects, which indicates that the specific spatial-frequency content of a stimulus type or its repetition had no effect on the CLB process. In a similar manner, level repetition improved overall performance, but did not reduce VF effects.

While these results suggested that the examined factors are unrelated to CLB we could not exclude that our null results were due to our specific method. Therefore, we conducted a second experiment with a procedure that was more similar to that in the study of Flevaris et al. (2010). In that experiment participants had to identify an unmasked prime stimulus (hierarchical letter) before the masked main stimulus (hierarchical letter) was presented. Moreover, the prime stimulus appeared at the center of the display, so that both hemispheres were stimulated similarly. Finally, all relevant factors were blocked.

Indeed, the procedural modifications in Experiment 2 had substantial effects. When the target level repeated between prime task and main task, not only the overall rate of conjunction errors was reduced, but also the corresponding VF effects. Although it cannot be inferred which of the differences between the experiments was responsible for this effect, it is likely that using unmasked and centrally presented prime stimuli was crucial. In any case, this result indicates that, under appropriate conditions, CLB can be improved by target-level repetition.

In contrast to target-level repetition, stimulus-type repetition had again no effect. However, stimulus type modulated the VF effects in Experiment 2. Interestingly, compared to Experiment 1, the procedure prevented a global advantage and that the stimulus types differed in their relative level dominance. Nevertheless, the outlined stimuli produced substantial smaller VF effects for global and local processing than the filled stimuli. This indicates that CLB was easier for the outlined stimuli.

Together, our results demonstrate that CLB can be affected by various factors. But what do the results tell us about the underlying binding mechanism? One possible interpretation is that all effective factors modulated spatial-frequency selection, as proposed by Flevaris et al. (2010). Thus, the effects of stimulus type might be explained by assuming that, under the specific priming conditions in Experiment 2, spatial-frequency parsing was more difficult for filled stimuli than for outlined ones (cf. Ivry and Robertson, 1998), and that this was disadvantageous for CLB. Yet, we can only speculate why parsing was more difficult for filled stimuli. Perhaps their strong low spatial frequencies impaired the parsing process. Indeed, it has been proposed that low-spatial-frequency channels inhibit high-spatial-frequency channels (cf. Hughes et al., 1996). Thus, if the power of low spatial frequencies is high, the resulting inhibition could make spatial-frequency parsing relatively difficult. Alternatively, it could be that, because the masks had the same spatial-frequency characteristic as the stimuli, the filled mask had a more interruptive effect on the binding mechanism than the outlined mask. Because until now, only few data have been collected with the masking paradigm, further research is needed to decide which account is more plausible.

The result that CLB was improved by target-level repetition is also compatible with a spatial-frequency interpretation. Because spatial frequencies and stimulus levels are related (Ivry and Robertson, 1998) these two factors are usually confounded, which also holds for the present experiments. Consequently, it is generally difficult, if not impossible, to separate effects of target-level repetition from 
those of the corresponding spatial-frequency repetition, especially if relative spatial frequency is considered. Thus, one could assume that in our case the positive effects of level repetition on CLB were caused by spatial-frequency priming.

However, our result that CLB was improved by target-level repetition can also be explained by assuming that abstract level categories were primed. Moreover, it is even possible to reinterpret the results of Flevaris et al.'s (2010) accordingly, and to propose that their compound sinusoidal gratings were mentally also represented as a hierarchical structure and that, therefore, focusing to a certain spatial-frequency component actually meant focusing to a certain stimulus level. Further support for this interpretation is provided by a recent study in which these researchers have shown that priming also works in the opposite direction, i.e., from the levels of hierarchical letters to the spatial-frequency components of a compound sinusoidal grating (Flevaris et al., 2011).

It might be difficult to imagine that two superimposed sinusoidal gratings, which are obviously not related to each other by a part/whole relationship, are nevertheless represented as hierarchical object. However, "local" and "global" are broader categories than just "part" and "whole." This has been shown by Hübner and Studer (2009), who used images of patterned animals as stimuli. The task for the global level was to categorize the animal, whereas that for the local level was to categorize the pattern. Although the patterns were merely subordinate features of the animals and not a constituent part, the typical results of global/local processing were observed, including VF effects.

What is more plausible for explaining our results: the spatialfrequency or the abstract-level account? Instead of choosing the one or the other alternative, we propose to integrate both. For this objective it might be helpful to focus on the differences between spatial frequency and levels, rather than on their similarities. Even though the two concepts are related (Ivry and Robertson,

\section{REFERENCES}

Doricchi, F., and Incoccia, C. (1998). Seeing only the right half of the forest but cutting down all the trees? Nature 394, 75-78.

Flevaris, A.V., Bentin, S., and Robertson, L. C. (2010). Local or global? Attentional selection of spatial frequencies binds shapes to hierarchical levels. Psychol. Sci. 21, 424-431.

Flevaris, A. V., Bentin, S., and Robertson, L. C. (2011). Attention to hierarchical level influences attentional selection of spatial scale. J. Exp. Psychol. Hum. Percept. Perform. 37, 12-22.

Hübner, R. (1997). The effect of spatial frequency on global precedence and hemispheric differences. Percept. Psychophys. 59, 187-201.

Hübner, R. (2000). Attention shifting between global and local target levels: the persistence of level-repetition effects. Vis. Cogn. 7, 465-484.

Hübner, R., and Malinowski, P. (2002). The effect of response competition on functional hemispheric asymmetries for global/local processing. Percept. Psychophys. 64, 1290-1300.

Hübner, R., and Studer, T. (2009). Functional hemispheric differences for the categorization of global and local information in naturalistic stimuli. Brain Cogn. 69, 11-18.

Hübner, R., and Volberg, G. (2005). The integration of object levels and their content: a theory of global/local processing and related hemispheric differences. J. Exp. Psychol. Hum. Percept. Perform. 31, 520-541.

Hübner, R., Volberg, G., and Studer, T. (2007). Hemispheric differences for global/local processing in divided attention tasks: further evidence for the integration theory. Percept. Psychophys. 69, 413-421.

Hughes, H. C., Nozawa, G., and Kitterle, F. (1996). Global precedence, spatial frequency channels, and the statistics of natural images. J. Cogn. Neurosci. 8, 197-230.
1998), they are by no means interchangeable. The terms "global" and "local" denote rather abstract and discrete level categories for representing hierarchical objects, whereas the term "spatialfrequency" denotes a low-level and continuous physical property of visual stimuli. The abstract character of the level categories is the reason why letter identities and levels are represented independently at early stages of processing, as stated by the CLB theory. In contrast, after all we know it makes little sense to assume that identity and spatial frequencies of a letter are represented independently at early stages, because identification is based on the specific spatial frequencies.

However, the fact that spatial frequencies are low-level stimulus features makes them to ideal candidates for connecting letter identities and abstract levels of hierarchical stimuli. It is conceivable that for deciding whether a given identity belongs to the local or to the global level of a stimulus, the system checks whether the spatial frequencies that were used for identification fall into the one or into the other spatial-frequency range associated with the two levels. The efficiency of this differentiation between the respective spatial frequencies seems to depend on the specific stimulus type.

Thus, our results suggest that the levels of hierarchical letters are represented by abstract categories and that the letter identities are bound to these categories by means of their distinctive spatial frequencies. The efficiency of the binding process can be improved if the level categories are pre-activated by level repetition, and if the spatial frequencies ranges related to the levels can easily be separated. Although partly speculative, this view not only nicely accounts for the data in this and related studies, but also integrates the CLB theory (Hübner and Volberg, 2005) and the DFF theory (Ivry and Robertson, 1998).

\section{ACKNOWLEDGMENT}

We thank Michael Dambacher for his helpful comments.
Ivry, R. B., and Robertson, L. C. (1998). The Two Sides of Perception. Cambridge, MA: MIT Press.

Kim, N., Ivry, R. B., and Robertson, L. C. (1999). Sequential priming in hierarchically organized figures: effects of target level and target resolution. J. Exp. Psychol. Hum. Percept. Perform. 25, 715-729.

Lamb, M.R., andYund,E.W.(1996).Spatial frequency and attention: effects of level-, target-, and location-repetition on the processing of global and local forms. Percept. Psychophys. 58, 363-373.

Lamb, M. R., Yund, E. W., and Pond, H. M. (1999). Is attentional selection to different levels of hierarchical structure based on spatial frequency? J. Exp. Psychol. Gen. 128, 88-94.

Lovegrove, W. J., and Pepper, K. (1994) "The influence of low-level processing in the global precedence effect," in Cognitive Approches to Human Perception, ed. S. Ballesteros (Hillsdale: Lawrence Erlbaum), 71-90.
Malinowski, P., Hübner, R., Keil, A., and Gruber, T. (2002). The influence of response competition on cerebral asymmetries for processing hierarchical stimuli revealed by ERP recordings. Exp. Brain Res. 144, 136-139.

May, J. G., Gutierrez, C., and Harsin, C. A. (1995). The time-course of global precedence and consistency effects. Int. J. Neurosci. 80, 237-245.

Miller, J., and Navon, D. (2002). Global precedence and response activation: evidence from LRPs. Q. J. Exp. Psychol. A 55, 289-310.

Navon, D. (1977). Forest before the trees: the precedence of global features in visual perception. Cogn. Psychol. 9, 353-393.

Robertson, L. C. (1996). Attentional persistence for features of hierarchical patterns. J. Exp. Psychol. Gen. 125, 227-249.

Robertson, L. C., Egly, R., Lamb, M. R., and Kerth, L. (1993). Spatial attention and cuing to global and local 
levels of hierarchical structure. J. Exp. Psychol. Hum. Percept. Perform. 19, 471-487.

Treisman, A., and Gelade, G. (1980). A feature-integration theory of attention. Cogn. Psychol. 12, 97-136.

Treisman, A., and Schmidt, H. (1982). Illusory conjunctions in the perception of objects. Cogn. Psychol. 14, 107-141.

Van Kleeck, M. H. (1989). Hemispheric differences in global versus local processing of hierarchical visual stimuli by normal subjects: new data and a meta-analysis of previous studies. Neuropsychologia 27, 1165-1178.

Volberg, G., and Hübner, R. (2004). On the role of response conflicts and stimulus position for hemispheric differences in global/local processing: an ERP study. Neuropsychologia 42, 1805-1813.

Yovel, G., Yovel, I., and Levy, J. (2001). Hemispheric asymmetries for global and local visual perception: effects of stimulus and task factors. J. Exp. Psychol. Hum. Percept. Perform. 27, 1369-1385.
Conflict of Interest Statement: The authors declare that the research was conducted in the absence of any commercial or financial relationships that could be construed as a potential conflict of interest.

Received: 18 March 2011; accepted: 07 June 2011; published online: 20 June 2011.

Citation: Hübner R and Kruse R (2011) Effects of stimulus type and level repetition on content-level binding in global/local processing. Front. Psychology 2:134. doi: 10.3389/fpsyg.2011.00134

This article was submitted to Frontiers in Perception Science, a specialty of Frontiers in Psychology.

Copyright $\odot 2011$ Hübner and Kruse. This is an open-access article subject to a nonexclusive license between the authors and Frontiers Media SA, which permits use, distribution and reproduction in other forums, provided the original authors and source are credited and other Frontiers conditions are complied with. 